\title{
Neuromorphic Engineering Needs Closed-Loop Benchmarks
}

\author{
Moritz B. Milde*, Saeed Afshar, Ying Xu, Alexandre Marcireau, Damien Joubert, \\ Bharath Ramesh, Yeshwanth Bethi, Nicholas O. Ralph, Sami El Arja, Nik Dennler, \\ André van Schaik and Gregory Cohen*
}

International Centre for Neuromorphic Systems, The MARCS Institute for Brain, Behaviour, and Development, Western Sydney University, Penrith, NSW, Australia

Neuromorphic engineering aims to build (autonomous) systems by mimicking biological systems. It is motivated by the observation that biological organisms-from algae to primates-excel in sensing their environment, reacting promptly to their perils and opportunities. Furthermore, they do so more resiliently than our most advanced machines, at a fraction of the power consumption. It follows that the performance of neuromorphic systems should be evaluated in terms of real-time operation, power

OPEN ACCESS

Edited by:

Timothy K. Horiuchi, University of Maryland, College Park,

United States

Reviewed by:

Qinru Qiu,

Syracuse University, United States Ulrich Rükert,

Bielefeld University, Germany

*Correspondence: Moritz B. Milde moritz.milde@gmail.com Gregory Cohen

g.cohen@westernsydney.edu.au

Specialty section:

This article was submitted to Neuromorphic Engineering, a section of the journal

Frontiers in Neuroscience

Received: 11 November 2021

Accepted: 24 January 2022

Published: 14 February 2022

Citation:

Milde MB, Afshar S, Xu Y, Marcireau A, Joubert $D$, Ramesh $B$, Bethi Y, Ralph NO, El Arja S,

Dennler N, van Schaik A and Cohen G (2022) Neuromorphic Engineering Needs Closed-Loop Benchmarks.

Front. Neurosci. 16:813555. doi: 10.3389/fnins.2022.813555 consumption, and resiliency to real-world perturbations and noise using task-relevant evaluation metrics. Yet, following in the footsteps of conventional machine learning, most neuromorphic benchmarks rely on recorded datasets that foster sensing accuracy as the primary measure for performance. Sensing accuracy is but an arbitrary proxy for the actual system's goal-taking a good decision in a timely manner. Moreover, static datasets hinder our ability to study and compare closed-loop sensing and control strategies that are central to survival for biological organisms. This article makes the case for a renewed focus on closed-loop benchmarks involving real-world tasks. Such benchmarks will be crucial in developing and progressing neuromorphic Intelligence. The shift towards dynamic real-world benchmarking tasks should usher in richer, more resilient, and robust artificially intelligent systems in the future.

Keywords: neuromorphic engineering, benchmarks, event-based systems, DAVIS, DVS, ATIS, audio, olfaction

\section{INTRODUCTION}

Despite the significant strides made in neuromorphic engineering in recent years, the field has not yet seen widespread industrial or commercial adoption. There is clearly difficulty in translating the research output of the field into real-world and commercially successful applications. Neuromorphic engineering has individually demonstrated many significant and valuable concepts, evidenced by dedicated large-scale neuromorphic processors (Davies et al., 2018), power-efficient analogue neuron circuits (Chicca et al., 2014; Moradi et al., 2018), on-chip and local unsupervised learning circuitry (Qiao et al., 2015), scalable parallel message-passing architectures (Furber, 2016), and retina-inspired and compressed visual sensing (Lichtsteiner et al., 2008). There are also active research and commercialisation efforts in applications of this research, including in Event-based Space Situational Awareness (Cohen et al., 2019), autonomous vehicle sensors (Perot et al., 2020; Gehrig et al., 2021), and for home security monitoring (Park et al., 2019; Samsung, 2020). However, the field struggles to integrate, build upon, and convey these successes to the wider engineering and scientific community. 
This article examines potential reasons for this slow dissemination by assessing the role that datasets, benchmarking problems, and comparative metrics play in presenting neuromorphic engineering to existing scientific communities. Adhering to existing benchmarking metrics, designed for fundamentally different processing and sensing systems, may limit our ability to report and, perhaps more importantly, to describe the performance and advantages of neuromorphic systems. Additionally, the ubiquity of such metrics complicates the development of new approaches to tackling existing problems with novel solutions. This is especially prevalent when moving away from uniformly sampled sensing and synchronised processors.

Progress in conventional computer vision and machine learning has been built upon datasets and static problems. The most significant strides in computer vision and deep neural networks were spurred by the ImageNet moment (Krizhevsky et al., 2017) and the rise of data-driven systems (Torralba and Efros, 2011), leading to some truly astonishing capabilities, from the ability to achieve human-like (and even super-human) levels of performance under ideal viewing conditions on certain vision tasks (He et al., 2016; Geirhos et al., 2017), to the unsettling ability to realistically replace faces and people in highdefinition video (Wang et al., 2021). However, such cuttingedge data-driven systems require unprecedentedly large datasets that have only become feasible in terms of size and required computation starting with the release of ImageNet in 2012 and the advent of high-performance computing centres. These datadriven approaches are unlikely to scale with increasing task complexity. The corresponding networks ingesting this data have grown vast in size and scale. Large datasets become difficult to distribute and test against and even more difficult to collect. Only a handful of organisations possess the resources required to collect and generate the cutting-edge datasets used at the forefront of deep learning. Furthermore, immunity to variable and degraded viewing conditions are still a problem that static datasets do not tackle efficiently and closed-loop benchmarks are better suited to test these conditions.

Larger datasets have enabled researchers to train everlarger networks, but, importantly, have also provided a meaningful way to compare different algorithms and approaches. This has driven researchers to optimise and push the limits of the technologies and algorithms through a mutually understood and quantifiable way of measuring success. Novel datasets and benchmarks will not only push model and algorithmic complexity, but also implicitly advance our understanding of distributed, parallel, and even neural computation.

Neuromorphic engineering has naturally followed a similar trajectory, both through the conversion of existing datasets to a neuromorphic format (Orchard et al., 2015a) and through the collection and creation of new datasets (Perot et al., 2020; Gehrig et al., 2021). The growth of neuromorphic computing has further driven the need for suitable neuromorphic benchmarks to showcase the utility of its approaches to artificial intelligence. Similar to conventional machine learning, this demand has led to the rise and proliferation of static neuromorphic datasets and, similarly, these have been instrumental in the field's advancement and growth.

However, our paper will detail how these approaches may actually be constricting the ability of neuromorphic engineering to tackle real-world problems in novel ways using approaches that embody and showcase the unique benefits of a fundamentally different way of operation. We discuss the history of neuromorphic benchmarking (see Section 1.1) and highlight the advantages and implications of sensing and processing in the context of closed-loop control systems (see Section 2). We further provide an overview of existing open-loop datasets, discuss in greater detail their downsides (see Section 3), and then apply the same analysis towards existing open-loop neuromorphic benchmarks (see Section 3.1).

After a brief overview and discussion of existing closedloop conventional benchmarks (see Section 3.2) and simulation environments available to create new closed-loop benchmarks (see Section 3.3), we describe our efforts in designing and developing a new generation of physically embedded, closedloop neuromorphic benchmarks (see Section 4). We finish with concluding remarks for future developments of closed-loop benchmarks to bootstrap the next generation of artificial and neuromorphic intelligence.

\subsection{History of the Analysis of Neuromorphic Benchmarks}

The neuromorphic community has long recognised the importance of datasets and their potential limitations, which led to a special research topic in Frontiers in Neuroscience: Neuromorphic Engineering in 2015 devoted specifically to neuromorphic benchmarking and challenges ${ }^{1}$. The proposal for the topic describes a situation not dissimilar to the current state of neuromorphic engineering in terms of the ability to make meaningful and representative comparisons of neuromorphic systems, both to one another and conventional systems. The papers published in that research topic provided a thorough overview of the existing efforts to create benchmarking approaches and included papers focusing on domain-specific and modality-specific sets of requirements and needs.

In fact, Stewart et al. (2015) directly addressed the need for closed-loop benchmark tasks in neuromorphic engineering, describing a closed-loop benchmark as a two-way interaction task in which the "output of the neuromorphic hardware influences its own future input" (Stewart et al., 2015). Highlighting the challenges involved in providing closed-loop tasks in place of static datasets, the authors suggested that this can only be accomplished by either providing a fully-specified physical system or providing a software simulation of the system. Building upon these ideas, this article summarised the existing simulators and related problems, highlighting the shortcomings of simulators and the difficulty in translating these into realworld applications and strongly motivating the need for realworld physical systems.

In addition, Tan et al. (2015) provided a thorough summary of the efforts to benchmark neuromorphic vision systems and

\footnotetext{
${ }^{1}$ https://www.frontiersin.org/research-topics/3448/
} 
outlines some of the lessons learned in creating and using the available datasets. Core to the arguments, in this article, the authors introduce the problems encountered when using static images with neuromorphic vision sensors, highlighting that the type of static object recognition problems found in conventional computer vision has no direct parallel in biology and therefore are not a task that biological systems have evolved to tackle (Tan et al., 2015). Contributing to this point, this article also stresses that neuromorphic vision datasets should be as representative of the real-world as possible. As our paper seeks to motivate, the move to real-world benchmarking tasks will inherently solve this problem.

The discussion around the development of the PokerDVS and MNIST-DVS datasets by Serrano-Gotarredona and Linares-Barranco (2015) also provides valuable insight into the historical reasons contributing to the reliance on datasets in the neuromorphic community. They point to the difficulty in obtaining neuromorphic hardware as a driving factor in the production of datasets that allow researchers to explore neuromorphic techniques without having physical access to scarce hardware resources. Whilst the supply and dissemination of neuromorphic sensors has drastically improved, the point remains a valid one, and there is still a strong need for neuromorphic datasets to enable access to the technologies.

Beyond the historical perspective, the authors also point out that the original poker dataset required manual curating due to noise, card edges, and the numbers on the cards. Their automated tracking method struggled with these factors, requiring annotations by hand to produce correctly labelled data. This highlights both the difficulty in acquiring large volumes of labelled data, and the question of inadvertently injecting additional context into the problem through factors such as labelling bias.

\subsection{Promises of Neuromorphic Systems}

To overcome the limitations of existing neuromorphic benchmarks, we argue that performance of neuromorphic systems should directly be evaluated based on latency, power consumption, and task-specific control metrics, rather than on a plain and static accuracy metric. This move inherently requires closed-loop sensing and processing, which in turn favours highly recurrent and feedback-heavy algorithms and architectures. Predictive algorithms naturally result, since for an agent to make an informed decision and react appropriately in a given environment, the past and present estimates of the state of said environment hardly matter. What does matter is the agent's expectation of future states, i.e., how the environment is going to change (Davies, 2019).

Closed-loop benchmarks require algorithms to holistically optimise for real-world constraints and power consumption while operating in real-time. Closed-loop benchmarks also require the ability to respond appropriately to ambiguous and partial inputs from an uncontrollable noisy and dynamic environment. Hence, we anticipate that designing algorithms for such tasks will lead to richer, more advanced, resilient, and truly intelligent artificial systems inspired by their biological counterparts. The physically embedded nature of biological processing, and the associated physical size, weight, power, and speed limitations that come with it, are fundamental aspects of the operation of such systems and cannot be treated as afterthoughts to be simulated or optimised in the final development phase.

Inspired by biological sensory-processing systems, the neuromorphic sensing and processing paradigm targets these requirements by providing resilient, parallel, asynchronous, and highly distributed sensory-processing solutions (Mead, 1990; Liu and Delbruck, 2010; Hamilton et al., 2014). The resulting neuromorphic processors are non-Von Neumann computing architectures that feature local learning mechanisms and are capable, when combined with neuromorphic sensors, of time-continuous, asynchronous and distributed information processing, with high power efficiency than their conventional clock-based counterparts (Thakur et al., 2019).

The gravitation of the neuromorphic community towards machine learning-like datasets is understandable, since the generation of alternative closed-loop datasets is at once challenging and very resource-intensive while simultaneously lacking the legitimacy of established large scale open-loop machine learning-like benchmarks (Grother, 1995; Jia Deng et al., 2009; Geiger et al., 2013; Xu et al., 2020). Novel embedded closed-loop benchmarks, however, will spur the development of closed-loop sensing, dynamic processing, and decision making systems, which is where neuromorphic computing has the greatest potential for providing advances in technology and computational models.

\section{DIFFERENT STYLES OF SENSING}

Sensors, irrespective of their sensing modality, can be classified into two distinct categories: passive sensors and active sensors. Passive sensor strategies do not emit energy into the environment when acquiring samples or data (see Figure 1, top row). A common example is found in autonomous systems, in which conventional image sensors employ a passive sensing approach to detect and process stimuli scattered by the immediate environment (Rees, 1990). In contrast, active sensors emit energy directly into their environment to elicit data, sampling a composition of the interactions of the actively emitted energy on the environment and any scattered energy already present in the environment (see Figure 1, bottom row). Autonomous systems may also employ active sensing regimes, such as RADAR and LiDAR, to parse their environment by instead varying sensor characteristics based upon the global state or by acting upon the immediate environment (Gini and Rangaswamy, 2008).

Sensor strategies can also be split by whether the control of the sensor is influenced by the output of the sensor. Moving the sensor in response to its output is also sometimes called active sensing ${ }^{2}$, but here we adopt the term closed-loop sensing for this mode of operation to avoid confusion, and open-loop sensing for the mode where the sensor output has no impact on the sensor itself. In open-loop systems, the sensor is simply a source of data for the rest of the system, allowing for very simple sensor

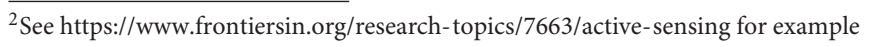




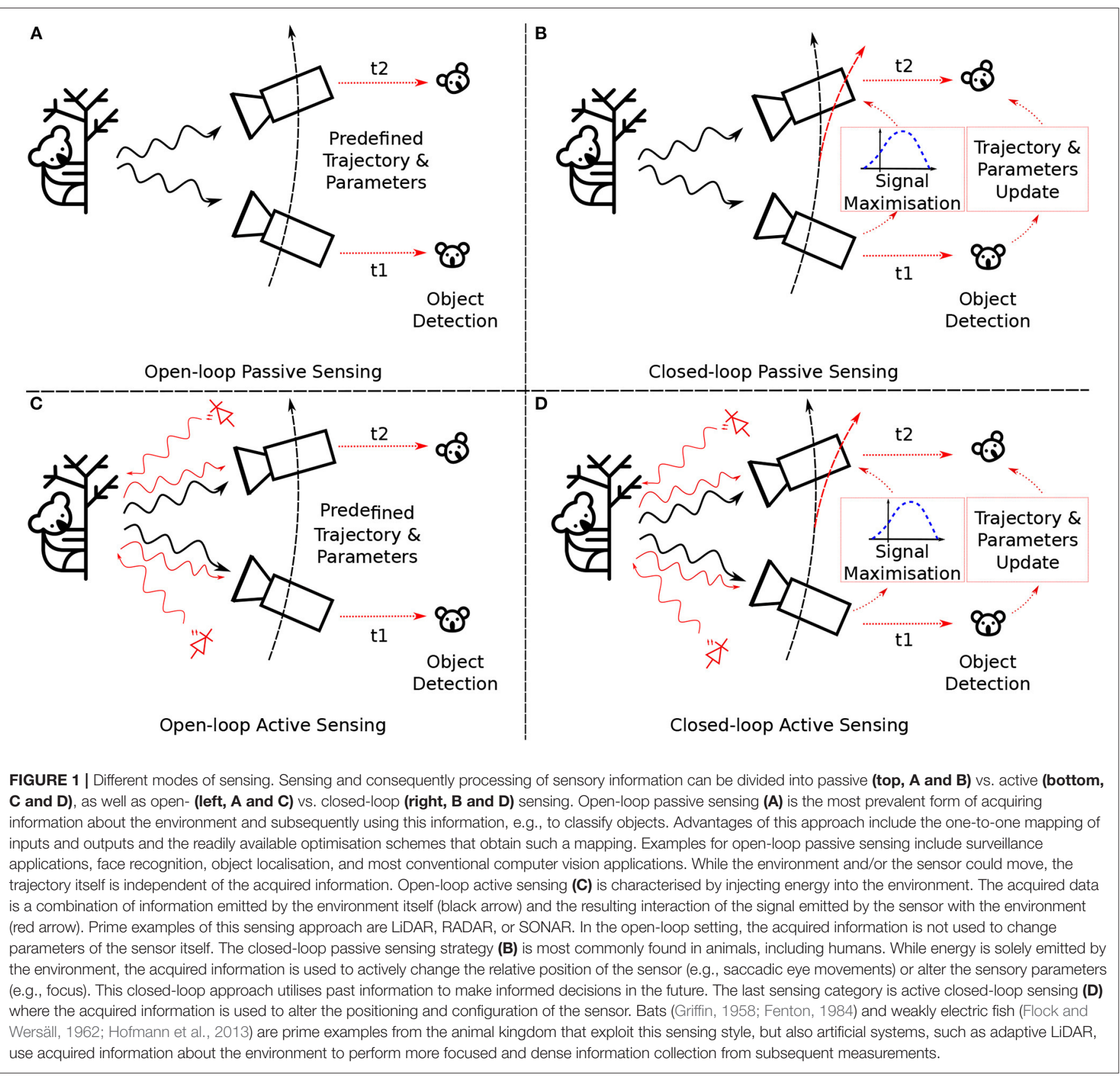

designs (see Figure 1, left column). Closed-loop systems integrate the sensor far more deeply into the system, and aspects of the sensor are actively modified as a function of its output to increase the relevant information in the sensor's output (see Figure 1, right column). Closed-loop systems are more complicated to design but offer the potential to extract far more task-relevant information from the sensor.

These two ways to categorise sensors are not mutually exclusive, and indeed there exist closed-and open-loop strategies for both active and passive systems (see Figure 2). The passive and active sensing strategies can both benefit greatly from a closed-loop methodology, especially when an internal model of the system is used to produce informed decisions to update sensor settings and model parameters. Practical examples of such systems include the closed-loop passive sensing techniques of stimulating contrast detection in event-based vision sensors with ego-motion, trading temporal resolution for spatial resolution (Yousefzadeh et al., 2018; D’Angelo et al., 2020).

Open-loop systems have the advantage of simplicity, in terms of their design and in terms of the data that they produce. By definition, open-loop sensing does not feature a feedback mechanism and therefore acquired samples have no effect on the next sample acquired by the sensor. As the sensor can be treated solely as a static source of information, recorded 


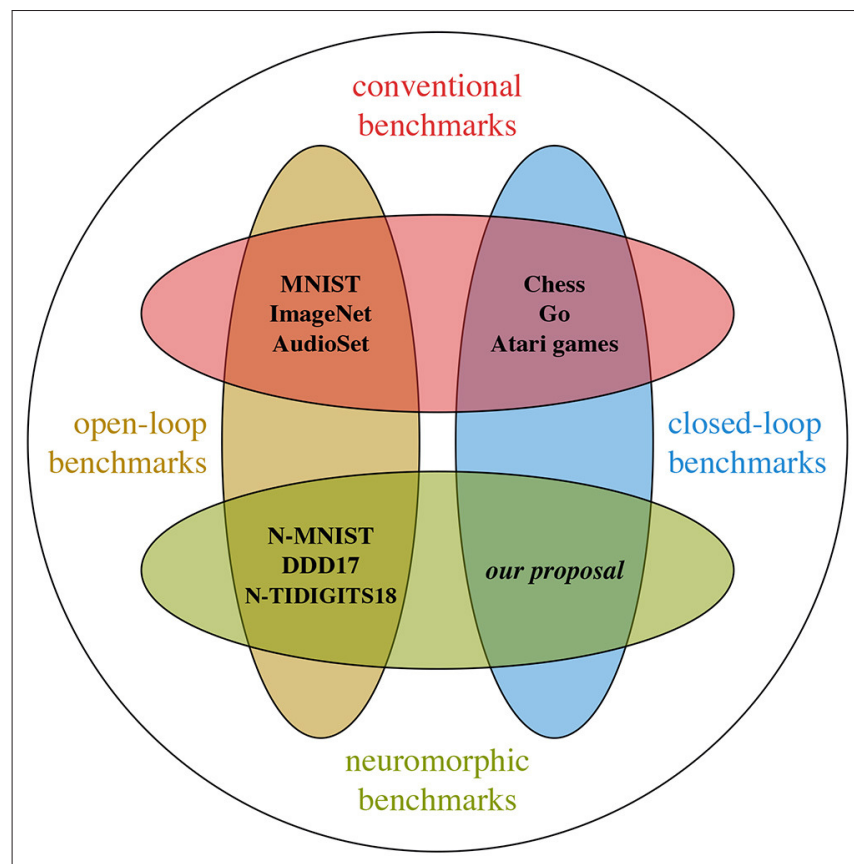

FIGURE 2 | Existing datasets and benchmarks fall into two categories: open-loop benchmarks, or datasets, and closed-loop benchmarks.

Supervised machine learning relies mostly on the first category, whereas reinforcement learning requires the second. Most existing neuromorphic engineering benchmarks fall in the first category. This article pleads in favour of closed-loop neuromorphic benchmarks.

datasets can easily be shared with the research community, allowing different algorithms to be compared without the need to replicate the interactions between the system and the sensor. This greatly simplifies the creation and the use of open-loop datasets, as no sensor state information needs to be known or stored. This simplicity, however, imposes limits on the nature of the problems being tackled. Problems are often carefully chosen, or restricted, to enable the use of an open-loop sensor (for a non-exhaustive list of existing open-loop datasets see Section 3). Such open-loop sensing approaches and their resulting datasets, however, limit the real-world applicability of an algorithm as information that could be beneficial to adjust to the environment is irreversible lost.

Systems for real-world problem solving such as autonomous driving (Bojarski et al., 2016) and process control (Firoozian, 2014) generally require algorithms with feedback mechanisms to proactively sample the environment and act accordingly. Potential feedback actions include changing the sensor position, the sensor configuration, or some aspect of the interface between the sensor and the environment. Adding a mechanism of feedback allows a system to observe the result of its interaction with the environment when solving compound real-world problems (Åström and Murray, 2010). With the inclusion of some element of (dynamic) memory capacity, these systems can be extended to achieve a degree of statefulness, using the recurrent nature of the system feedback to build an internal model of the surrounding environment (Rao and Ballard, 1999;
Friston, 2010; Rasmussen et al., 2017; Hogendoorn and Burkitt, 2018; Keller and Mrsic-Flogel, 2018).

The stateful memory capacity inherent in closed-loop systems is partially determined by the dimensionality of the feedforward signal, but primarily determined by the dimensionality of both feedback and recurrent pathways. Here, neuromorphic sensoryprocessing systems are of special interest due to their continuous and implicit representation of time in sensing and processing, thus increasing the resolution of their temporal dimension for all three information pathways (feedforward, feedback, and recurrent). This has the consequence, especially in closed-loop systems, that signals can be asynchronously distributed without the need for a centralised clock.

The path going forward towards machine intelligence, especially for neuromorphic technology, is not merely a substitution of neuromorphic sensors for conventional sensors, but instead, the creation of complete embedded systems that emulate the performance and constraints of their biologically inspired origins. To address this gap and to progress with closedloop benchmarking, we propose to build benchmarks that are physically embedded and require models operating in biological real-time. This approach provides the benchmark with an objective that inherently includes some form of decision making and action selection. These benchmarks would additionally feature sensory-motor systems that are subject to real-world fluctuations and noise, which the models would need to deal with.

\section{EXISTING BENCHMARKS}

The development of engineering systems, whether neuromorphic or otherwise, is driven by empirical studies on specific tasks or problems. The quality of a solution is measured with a benchmark-that is, a well-defined task associated with a test. The test yields a numerical score which can be used to compare solutions.

Complex problems in science and engineering are usually split into smaller ones; the so-called divide-and-conquer approach (Dustdar et al., 2020). Accordingly, benchmarks are generally designed for specific sub-problems rather than realworld tasks, with the underlying assumption that solving subproblems is integral to tackling real-world tasks. Datasets are a simple yet effective way to implement this strategy. Labelled real-world data makes for a reasonably neutral ground truth, which can be used to estimate an algorithm's accuracy, i.e., the distance to the ground truth, with respect to an agreed upon metric. This approach yields well-defined evaluation standards that facilitate comparison between methods, and encourage competition between researchers. For example, the NIST database (Grother, 1995) provides an objective measure of individual character recognition as a means to tackle handwriting recognition. It also serves as a good entry point for more complex machine learning problems (LeCun et al., 1998). Being valid representatives of a broader class of useful problems is a sought after feature for sub-problems (Davies, 2019).

Unfortunately, the divide-and-conquer approach has several shortcomings hindering our ability to design neuromorphic 
systems that tackle real-world tasks. First, tackling subproblems marginalises concerns that are only meaningful when considering real-world systems, notably power consumption and latency. It also encourages accuracy maximisation in arbitrary parts of the system, even if that accuracy may not be needed to solve the associated real-world task.

As far as neuromorphic engineering is concerned, the vast majority of existing benchmarks are open-loop (see Section 3.1 for critical review). Thus, there is no standard way to evaluate a closed-loop neuromorphic system's performance, latency or power consumption, even though neuromorphic engineering is well-suited to the design of such systems (Stewart et al., 2015).

Datasets are not the only type of benchmark. The Reinforcement Learning (RL) community relies on (simple) tasks that encompass both perception and action, such as Chess (Silver et al., 2018), Atari games (Mnih et al., 2013), or Go (Silver et al., 2017). The task itself is used as benchmark, therefore, the score is directly related to the intended outcome of the system, rather than being an arbitrary proxy (see Section 3.2 for short review). Much like conventional openloop benchmarks, existing closed-loop benchmarks cannot be used directly by neuromorphic engineering. The sensing modalities are fundamentally incompatible, and noise-free data is not representative of the output of neuromorphic sensors. Nevertheless, using simple yet complete problems as benchmarks is an idea that can be translated to neuromorphic engineering. Figure 3 illustrates our view of the current situation and shows that closed-loop neuromorphic benchmarks are heavily underrepresented.

\subsection{Neuromorphic Open-Loop Datasets}

The fundamental difference between conventional sensors and neuromorphic event-based sensors is in the way the signal of interest is sampled. While the former sampling approach uses discrete and fixed time intervals to synchronously sample the signal of interest, i.e., Riemann sampling (Åström and Bernhardsson, 2002), the latter approach uses only the relative change in signal amplitude to trigger the asynchronously reporting of events, i.e., Lebesque sampling ( $\AA$ ström and Bernhardsson, 2002).

To still be able to utilise the tremendous effort invested by the machine learning and machine intelligence community to construct open-loop datasets, they would need to be converted to comply with neuromorphic sensory-processing systems. In order to convert existing frame-based open-loop datasets into a spike- or event-based one, the pixel intensities, in case of a vision dataset, are used to calculate a Poisson distributed spike train (Orchard et al., 2015b; Cohen et al., 2018), or to calculate the time-to-first spike (Masquelier, 2012).

Alternatively, event-based sensors have been used directly to recreate existing open-loop datasets for handwritten digit recognition (Diehl and Cook, 2015; Orchard et al., 2015a; Cohen et al., 2018), object classification (Orchard et al., 2015a; Serrano-Gotarredona and Linares-Barranco, 2015; Cohen et al., 2018), autonomous driving (Binas et al., 2017; $\mathrm{Hu}$ et al., 2020), pedestrian detection (Miao et al., 2019), pose estimation (Mueggler et al., 2017; Calabrese et al., 2019), spoken digit classification (Anumula et al., 2018), or speaker identification (Ceolini et al., 2020) (please refer to Figure 3 or Tables 1, 2 for a more complete listing of existing datasets and benchmarks).

\subsection{Conventional Closed-Loop Benchmarks}

In closed-loop systems, contrary to open-loop ones, a sensor or agent is continuously receiving sensory stimuli from the environment (either time-varying or time-continuous). This sensory information is processed and ultimately used to either select an action or provide motor command signals that manipulate the environment or move the agent/sensor within it. Closed-loop interaction with the environment, as used in RL, alleviates the need to collect and hand-annotate large amount of data, as the agent learns online and based on partial information (Shalev-Shwartz, 2011) to maximise a reward.

The OpenAI gym environments (Brockman et al., 2016) provide a rich collection of curated closed-loop environments such as Atari games ${ }^{3}$, and continuous control tasks for robotic applications ${ }^{4}$. The OpenSim-RL environment provides the user with a biomechanics environment ${ }^{5}$ (Akimov, 2020), with the goal being to control a human body to accomplish diverse locomotion tasks such as arm movements or different gait patterns.

Simulated closed-loop systems have, however, witnessed their biggest and maybe most popular breakthrough with the release of alphaGo (Silver et al., 2017), which beat the leading world champion in the game of Go. This was followed by alphaZero (Silver et al., 2018) and alphaStar (Vinyals et al., 2019) beating their respective leading world champion in chess, shogi, and most impressively Starcraft. The game of Go is known for its untraceable decision tree while Starcraft is a competitive online multi-player real-time strategy computer game, making the RL capabilities of these Deepmind engines truly impressive. What needs to be considered here, though, is that these engines were operating directly on machine-level code rather than through a layer of visual or motor abstraction, enabling them to operate far faster than biological real-time, without any added sensory-motor noise.

Similar approaches have been used to artificially master other games such as Mario, Quake III (Jaderberg et al., 2019), Dota 2 (OpenAI: et al., 2019), or a host of Atari games (Badia et al., 2020).

\subsection{Simulators}

Simulators play an important role in lowering the barriers to interaction with otherwise expensive or complicated hardware and can greatly aid the exploration and prototyping of new and novel neuromorphic hardware. Simulation can be applied directly to neuromorphic sensors and computing hardware, which can in turn be used to develop, test, and even characterise neuromorphic algorithms and approaches. Simulation also allows for the exploration of situations, scenarios,

\footnotetext{
${ }^{3}$ https://gym.openai.com/envs/\#atari

${ }^{4}$ https://gym.openai.com/envs/\#mujoco

${ }^{5}$ http://osim-rl.stanford.edu/
} 


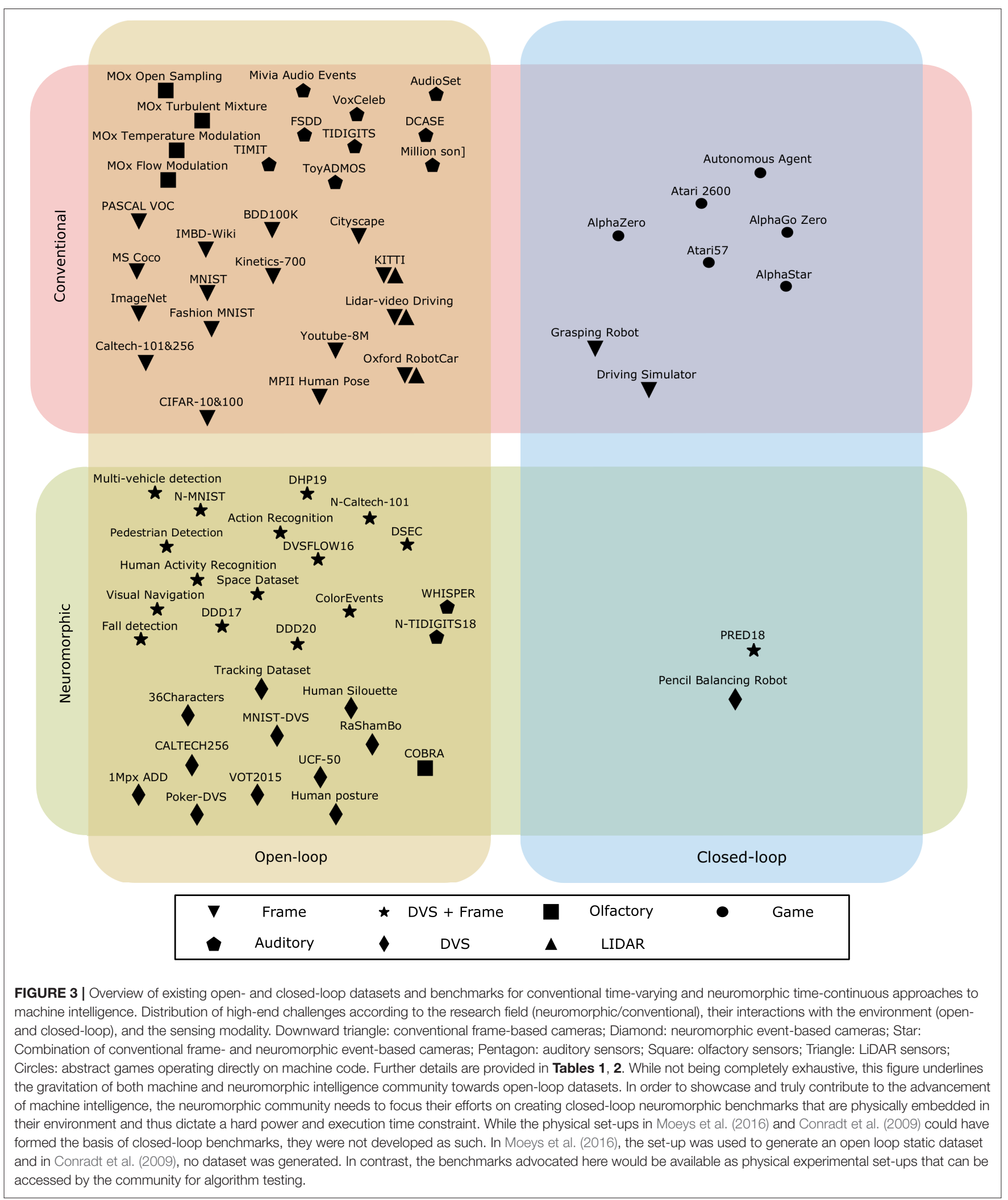


TABLE 1 | Conventional Benchmark Datasets for various sensor modalities.

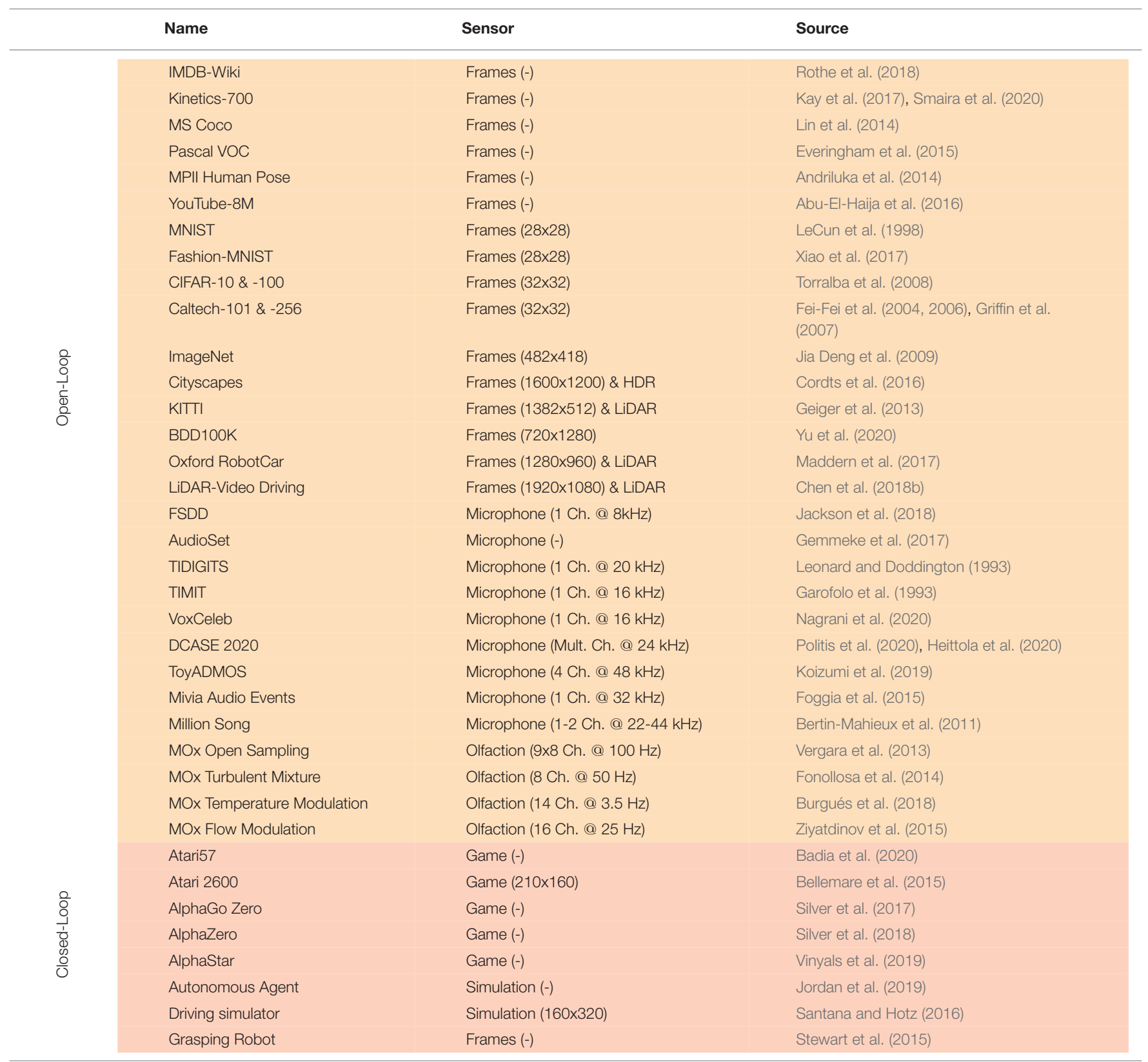

and environments that may be prohibitively difficult or pose technical challenges for real-world hardware.

Simulation techniques are already widely used in neuromorphic engineering. For example, simulation has been used to optimise existing event-based pixel designs (Remy, 2019) and to analyse and predict bottle-neck effects (Yang et al., 2017). Simulation can also allow for the rapid exploration of a vast number of potential scenarios, such as those found in real-world environments, and which would be impossible to physically test individually. Complex and hazardous scenarios are also expensive to emulate: for example, the pre-crash scenario used in designing of automotive vehicles can be tested with fake targets, but this restrains the evaluation to a single, very specific scenario, making the optimisation easy and leading to the issue of over-fitting (Segata and Cigno, 2019). Simulations enable us to explore a broader range of configurations in which there is direct access to the ground truth. This can also be used to augment and extend real-world datasets, such as for example Virtual KITTI (Gaidon et al., 2016) which extends the KITTI dataset (Geiger et al., 2013) to include simulated data for extreme driving conditions.

Simulators also enable the rapid exploration of the benefits offered by neuromorphic sensing when compared to conventional strategies, especially in cutting-edge challenges 
TABLE 2 | Neuromorphic Benchmark Datasets for various sensor modalities.

\begin{tabular}{|c|c|c|c|}
\hline & Name & Sensor & Source \\
\hline \multirow{29}{*}{$\begin{array}{l}\text { O } \\
0 \\
\frac{1}{1} \\
\frac{1}{8} \\
0 \\
00 \\
\frac{0}{0}\end{array}$} & Pedestrian detection & DAVIS 346 (346x240) & Miao et al. (2019) \\
\hline & Space Dataset & DAVIS 240 (240x180) \& ATIS (304x240) & Cohen et al. (2019) \\
\hline & DVSFLOW16 & DAVIS 240 (240x180) & Rueckauer and Delbruck (2016) \\
\hline & Visual navigation & DAVIS 240 C (240x180) & Barranco et al. (2016) \\
\hline & Action recognition & DAVIS 346 (346x240) & Miao et al. (2019) \\
\hline & Multi-vehicle detection & DAVIS 346 (346x240) & Chen et al. (2018a) \\
\hline & DHP19 & DAVIS 346 (346x240) & Calabrese et al. (2019) \\
\hline & Fall detection & DAVIS 346 (346x240) & Miao et al. (2019) \\
\hline & DDD17 & DAVIS 346 B (346×240) & Binas et al. (2017) \\
\hline & DDD20 & DAVIS 346 B (346×240) & Hu et al. (2020) \\
\hline & ColorEvents & ColorDAVIS 346 (346×240) & Scheerlinck et al. (2019) \\
\hline & $1 \mathrm{Mpx}$ Automotive Detection Dataset & High-resolution EBC (Finateu et al., 2020) (1280-720) & Perot et al. (2020) \\
\hline & DSEC & PPS3MVCD (640×480) & Gehrig et al. (2021) \\
\hline & RaShamBo & DVS (64x64) & Lungu et al. (2017) \\
\hline & 36Characters & DVS (128x128) & Orchard et al. (2015b) \\
\hline & MNIST-DVS & DVS (128x128) & $\begin{array}{l}\text { Serrano-Gotarredona and } \\
\text { Linares-Barranco (2015) }\end{array}$ \\
\hline & Poker-DVS & DVS (128x128) & $\begin{array}{l}\text { Serrano-Gotarredona and } \\
\text { Linares-Barranco (2015) }\end{array}$ \\
\hline & VOT2015 & DVS (128x128) & Hu et al. (2016) \\
\hline & Tracking Dataset & DVS (128x128) & Hu et al. (2016) \\
\hline & UCF-50 & DVS (128x128) & Hu et al. (2016) \\
\hline & CALTECH256 & DVS (128x128) & Hu et al. (2016) \\
\hline & Human silhouette & DVS (128x128) & Pérez-Carrasco et al. (2013) \\
\hline & Human posture & DVS (128x128) & Zhao et al. (2015) \\
\hline & N-Caltech101 & ATIS (304×240) & Orchard et al. (2015a) \\
\hline & N-MNIST & ATIS (304x240) & Orchard et al. (2015a) \\
\hline & Human activity recognition & ATIS (346x240) & Pradhan et al. (2019) \\
\hline & N-TIDIGITS18 & DAS $(64 \times 2 \times 4)$ & Anumula et al. (2018) \\
\hline & WHISPER & Microphone (16) & Ceolini et al. (2020) \\
\hline & COBRA & Olfaction (-) & $\begin{array}{l}\text { Schneider and Schneider (2003), } \\
\text { Schmuker and Schneider (2007) }\end{array}$ \\
\hline & PRED18 & DAVIS240C (240x180) & Moeys et al. (2016) \\
\hline $\begin{array}{l}0 \\
0 \\
0 \\
\frac{1}{1} \\
\frac{1}{8} \\
\mathscr{0} \\
\frac{0}{0}\end{array}$ & Pencil Balancing Robot & DVS (128x128) & Conradt et al. (2009) \\
\hline
\end{tabular}

such as drone racing (Madaan et al., 2020) or pose estimation (Mueggler et al., 2017). Simulation further eliminates the need to calibrate several real sensors, which is itself a challenging and open question. Uncalibrated and uncharacterised sensors can add temporal and spatial errors through different acquisition speeds and unsynchronised clocks (Zhu et al., 2018).

Some simulators, such as Carla (Dosovitskiy et al., 2017), take advantage of highly sophisticated rendering engines developed and optimised for the gaming industry. These tools have been extended and adapted to emulate neuromorphic vision sensors and have been successfully used to simulate data for a number of challenging tasks. An early example of such an application was a simulated driving task in which the algorithm must control a robotic car and keep it on the road (Kaiser et al., 2017). As part of the Neurobotics project ${ }^{6}$ it allows for the development of bio-inspired robots through simulations (Falotico et al., 2017). The project was built upon the Robotics Operation System (ROS) tool-chain (Quigley et al., 2009) and used a simulator known as Gazebo (Koenig and Howard, 2004), which emulates an eventdriven pixel using rendered images discretised in time. This was followed by Event SIMulator (ESIM), which is perhaps the most widely used event-based vision simulator in the neuromorphic community (Mueggler et al., 2017; Rebecq et al., 2018). It provides a simulation of a more realistic pixel behaviour and

${ }^{6}$ https://neurorobotics.net 
implements a novel method to adapt the time resolution of the rendering as a function of the dynamics of the scene. It has been used to create annotated datasets (Rebecq et al., 2019), or to simulate novel pixel designs with multi-spectral sensitivities (Scheerlinck et al., 2019). More recently, we have developed an even more realistic event based vision sensor simulator (Joubert et al., 2021), which has been used to simulate characterising the materials on resident space objects with event based sensors (Jolley et al., 2021).

In the past, simulation models of event-based sensors have been used to extend computer vision open-loop datasets like classification (Gehrig et al., 2020) and as a means of converting conventional datasets to event-based ones (Gehrig et al., 2020). Whilst this approach has merits, it faces inherent limitations when applied for event-based vision systems as the high temporal resolution, a hallmark of event-based sensing, is artificially interpolated and subject to quantisation errors. The different sources of noise are also neglected, and this loss of information might be detrimental to building fully real-world applicable systems. Finally, some limitations remain as no simulator perfectly replicates the real world, and the quantity-quality tradeoff of generated data, e.g., with respect to the level of detail in the simulation of the laws of physics, remains one of many unresolved limitations (Hu et al., 2021).

One of the most significant problems encountered with simulations relates to the often vast difference in difficulty between controlling a simulation and a physical system (Jakobi et al., 1995), with the main differences arising from the degree and nature of noise in the real-world system. We argue that this noise is not only inherent in neuromorphic systems, but perhaps even necessary to build functioning and robust algorithms and systems (Liang and Indiveri, 2019; Milde, 2019). The nature of noise in neuromorphic (and potentially biological systems) may be fundamentally different to how it is treated in conventional sensors and processing. Our efforts to mitigate this noise, either through post-processing or by designing systems that better approximate our idealised simulations, may have hindered our ability to deliver on the promises of neuromorphic algorithms and systems.

\section{NOVEL NEUROMORPHIC CLOSED-LOOP BENCHMARKS}

To close the gap between perfect simulations of the world and the imperfect reality we need to explore novel ways of building physically embedded closed-loop benchmarks and thus generate realistic training environments. This step towards closed-loop benchmarks will also spur and require the development of novel models of, and approaches to, machine and neuromorphic intelligence.

\subsection{Looking Beyond Accuracy as a Single Benchmarking Metric}

Accuracy is generally evaluated by calculating the difference between a desired high-level concept target (i.e., true object category) and the output of the model (i.e., the inferred object category). Accuracy alone does not encapsulate all performance metrics important in a real-world system. For example, closed-loop systems can have hard limitations placed on their response time, but the latency required to operate successfully is not captured by measures of performance accuracy. In order to address these restrictions we need to evaluate models beyond accuracy as a single benchmarking metric.

The majority of approaches to formulating an evaluation metric exclude training and execution time from the loss function and thus from the performance evaluation (Torralba and Efros, 2011). Similarly, power consumption, throughput and operations performed per unit time are not considered (Torralba and Efros, 2011). In addition there are other system evaluation metrics, such as racial or gender recognition biases or resiliency to adversarial attacks (Stock and Cisse, 2018).

Here, we propose to include these constraints implicitly in the benchmark's evaluation metric. Thus, the objective of a model competing on a physically embedded benchmark becomes to achieve the highest score with limited power consumption, unbiased data collection, limited throughput and a hard time constraint to react in biological or task-dependent real-time. This paradigm shift will spur the development of models which focus on closed-loop and predictive sensing and processing (Rao and Ballard, 1999; Moeys et al., 2016; Keller and Mrsic-Flogel, 2018), exploit spatio-temporal sparsity (Aimar et al., 2019) and are suited for novel real-time performing neuromorphic processing systems (Milde et al., 2017).

\subsection{A Case Study for Event-Based Closed-Loop Benchmarks}

Building on the needs and requirements identified for neuromorphic benchmarking systems, we have developed a set of required characteristics that are essential for creating benchmarking tasks that properly assess and quantify the performance of neuromorphic systems. These are:

Evaluation Metrics: The experimental setup should be capable of collecting critical information, such as power consumption and task performance, which is needed to evaluate the models.

Closed-loop embodiment: The benchmarking task should require at least one level of feedback. Therefore, the output, whether originating from early, intermediate, or late processing stages of the model should affect the input to the model, for example, by altering either perceptual parameters of the sensor, or the relative positioning of the agent and its sensors with respect to the environment.

Complexity: The environments should reflect the complexity that an agent can encounter in real-world scenarios and therefore include multiple possible states. The presence of noise, occlusions, and background clutter (or the equivalent noise and distractors in non-visual tasks) needs to be part of the environment if we desire to develop processing algorithms that are resilient to such effects. It is also important that the 
same environment be available for both the training and the testing environment.

Accessibility and Latency: The benchmarking task needs to be remotely accessible and have a clearly defined Application Programming Interface (API) to enable testing of different algorithms. The API should be capable of relaying and recording all the essential information from the experimental setup to the model and vice versa. The API needs to be opensource for transparency and needs to support different existing conventional and neuromorphic architectures. The API needs to operate at high-speed, with low latency, to allow algorithms to take full advantage of neuromorphic sensory-processing systems.

Replicability: The dynamics of the environment need to be able to be replicated. The experimental setup should be reliable enough to handle long trials and multiple runs with minimal deviation in performance. The setup has to sustain its behaviour over very long periods and produce reliable and repeatable results. As closed-loop benchmarks must evaluate applied algorithms in a consistent unbiased manner, they must, by necessity, exclude non-reproducible physical systems with non-ergodic behaviour.

The final point in this list implies that ideal closed-loop benchmarks cannot contain humans in the loop. However, a system which supports both, robot vs. robot and robot vs. human interaction, can be very interesting as the human opponent represents a source of noise which is informed but neither unbiased nor consistent. The introduction of a human opponent will also help in engaging non-experts in the discussion on the implications of research for the general public and will make it easier to convey the scientific efforts similarly to DeepMind's efforts with alphaGo.

To better define what such a novel, physically embedded closed-loop benchmark could look like, we will describe in the remainder of this article our efforts towards building a robotic foosball table. We started the design and development of the first iteration of a robotic foosball table for the 2019 Telluride Neuromorphic Engineering Workshop ${ }^{7}$. The idea was simply that, if a human or algorithm can beat another human or artificial opponent, i.e., score more goals in a game, the winner is better at the task of table foosball, giving us a straightforward performance metric (see Figure 4).

The setup was a standard foosball table with one side controlled by the machine and the other side open for human play. A strip of non-flickering LEDs illuminated the surface of the table. The ball had no special markers on its surface, to help in differentiating it from other movements on the table. A neuromorphic event-based camera (Brandli et al., 2014) was mounted on top of the table looking directly down towards the table surface providing both regular sampled frames and asynchronously sampled events. Neuromorphic vision sensors are exquisite at picking up fast-moving objects against a stationary background, but the dynamic motion of the player rods by both contestants provides many distractions by obstructing the ball below them.

$\overline{{ }^{7} \mathrm{http}: / / \text { tellurideneuromorphic.org }}$
The machine had eight degrees of freedom to control the translational and rotational movements of the four rods on the machine side. The mechanics were developed to ensure fast movement of the players with low latency to match the speed of the ball. One way to interact with the environment was through direct access to the eight motors controlling the rods via a microcontroller, but a more abstract and simple level of control was provided by controlling the position of the players on the table.

The problem of building a table foosball controller can be approached in multiple ways; it can be treated as a compound task of tracking and decision making, or as an end-to-end reinforcement learning task. The fast and dynamic environment demands algorithms which are capable of real-time processing of the events from the (neuromorphic) vision sensor. Thus, the benchmark intrinsically requires real-time predictive inference for successful gameplay and greatly benefits from non-batched, online and continuous learning approaches. The reason for this is simple: if one wants to hit a ball it is of negligible importance where the ball has been in the past, it hardly matters where the ball is right now, but it truly matters where the ball is going to be when one wants to hit it.

In this system, the performance evaluation can mainly be the game score and power consumption. On the foosball system we propose, the power consumption is constraint by the hardware we make available, but the computational demands of different algorithms will still impact the effective power consumption of the system. Also, the robotic foosball table as a benchmark could be copied in different locations and could use different hardware with different power consumption limitations. Such game score driven evaluation has been sufficient for developing systems such as alphaGo (Silver et al., 2018), but for human designed algorithm development, additional feedback will be required. For this purpose, recordings of the system made with the neuromorphic event-based camera will be made available to the researcher.

The current prototype iteration of the robotic foosball table is not yet ideal as a benchmark for neuromorphic algorithms. In an ideal scenario, both sides should be controlled by an algorithm or network and the winner remains and can be contested by another algorithm or network. We are currently developing such a table, where both sides can be controlled by a robotic system as well as a software stack for allowing remote access of the benchmark through a web-based API. We expect this foosball setup will pose a good first benchmark for conventional and neuromorphic algorithms to test their capabilities in a closed-loop setting.

\section{CONCLUDING REMARKS}

In this article, we discussed the understandable reasons why the research community, whether neuromorphic or not, gravitates towards open-loop datasets to train and evaluate their artificially intelligent algorithms or networks. While models and hardware accelerators are being developed to ensure operational real-time performance during inference on such open-loop datasets, online training within a limited time and power budget is being neglected in these solutions. 


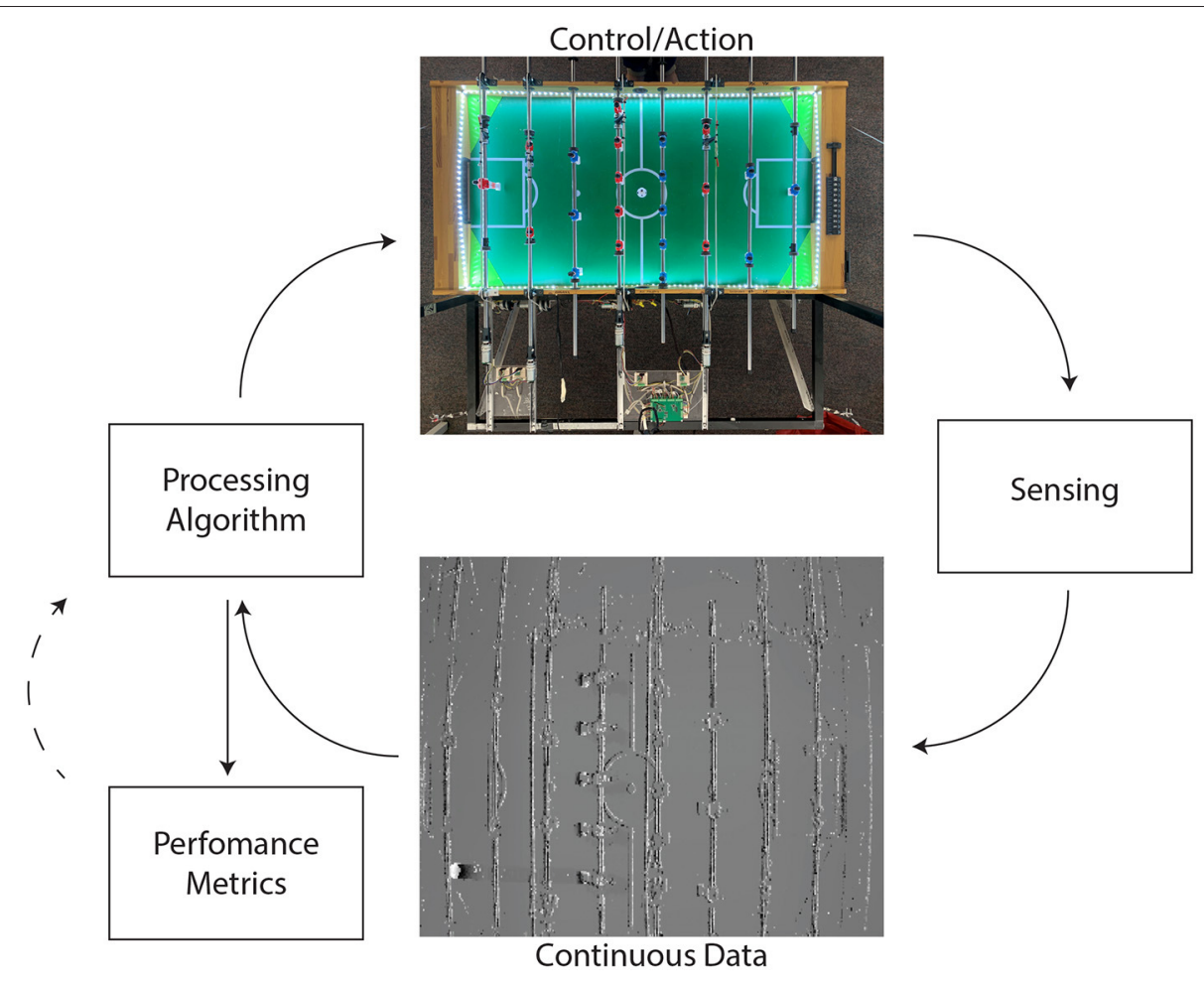

FIGURE 4 | Schematic of the closed-loop robotic foosball setup.

Alternatively, the closed-loop nature of Reinforcement Learning (RL) introduces a notion of online learning and decision making in models of machine intelligence. Conventional $\mathrm{RL}$ approaches introduce the requirement for operational real-time performance in inference, but not in training, nor do they address the issue of power consumption in their evaluation metrics. It appears that in most cases, the power consumption and real-time performance of both training and inference in models of machine intelligence are treated as afterthoughts, to be optimised afterwards using dedicated hardware accelerators or application-specific integrated circuit solutions.

The neuromorphic community has greatly benefited from the vast number of open-loop datasets and has often recreated and converted them for use in training neuromorphic algorithms and neural networks. However, the same is not true for closed-loop benchmarks, even though such benchmarks would play to the strengths of neuromorphic sensory-processing systems, i.e., low power consumption, high temporal resolution, distributed and local learning, robustness to noise, resilient processing due to parallel and redundant information processing pathways, and online unsupervised learning. The very essence of the eventbased sensing and computing paradigm, that time represents itself, should enable neuromorphic algorithms and spiking neural networks to naturally implement feedback control loops in which time and its continuous representation can act as the unifying entity for perception, learning, and action. The neuromorphic community is, however, missing benchmark tasks that require recurrent and feedback heavy algorithms and networks. To enable testing this assumption, we described our efforts in building a closed-loop, physically embedded robotic foosball system to function as a benchmark. We expect that robotic foosball, or similar physically embedded closedloop benchmarks, will be a crucial ingredient in advancing machine and neuromorphic intelligence to include the ability to perform time critical, informed decisions in noisy, ambiguous environments based on often partial information.

\section{AUTHOR CONTRIBUTIONS}

All authors listed have made a substantial, direct, and intellectual contribution to the work and approved it for publication.

\section{FUNDING}

This project was funded by Western Sydney University's Strategic Research Initiative. Some of the authors were supported by AFOSR grant FA9550-18-1-0471. 


\section{REFERENCES}

Åström, K. J. and Bernhardsson, B. M. (2002). "Comparison of Riemann and Lebesgue sampling for first order stochastic systems," in Proceedings of the IEEE Conference on Decision and Control, Vol. 2 (Las Vegas, NV: IEEE), 2011-2016.

Åström, K. J. and Murray, R. M. (2010). Feedback Systems: An Introduction for Scientists and Engineers. Princeton, NJ: Princeton University Press.

Abu-El-Haija, S., Kothari, N., Lee, J., Natsev, P., Toderici, G., Varadarajan, B., et al. (2016). YouTube-8M: a large-scale video classification benchmark. arXiv preprint arXiv:1609.08675.

Aimar, A., Mostafa, H., Calabrese, E., Rios-Navarro, A., Tapiador-Morales, R., Lungu, I. A., et al. (2019). NullHop: a flexible convolutional neural network accelerator based on sparse representations of feature maps. IEEE Trans. Neural Netw. Learn. Syst. 30, 644-656. doi: 10.1109/TNNLS.2018.2852335

Akimov, D. (2020). Distributed soft actor-critic with multivariate reward representation and knowledge distillation. arXiv preprint arXiv:1911.13056.

Andriluka, M., Pishchulin, L., Gehler, P., and Schiele, B. (2014). "2D human pose estimation: new benchmark and state of the art analysis," in Proceedings of the IEEE Computer Society Conference on Computer Vision and Pattern Recognition (Columbus, OH), 3686-3693.

Anumula, J., Neil, D., Delbruck, T., and Liu, S. C. (2018). Feature representations for neuromorphic audio spike streams. Front. Neurosci. 12:23. doi: 10.3389/fnins.2018.00023

Badia, A. P., Piot, B., Kapturowski, S., Sprechmann, P., Vitvitskyi, A., Guo, D., et al. (2020). "Agent57: outperforming the atari human benchmark," in 37th International Conference on Machine Learning, ICML 2020, PartF168147-1, 484-494.

Barranco, F., Fermuller, C., Aloimonos, Y., and Delbruck, T. (2016). A dataset for visual navigation with neuromorphic methods. Front. Neurosci. 10:49. doi: $10.3389 /$ fnins. 2016.00049

Bellemare, M. G., Naddaf, Y., Veness, J., and Bowling, M. (2015). "The arcade learning environment: an evaluation platform for general agents," in IJCAI International Joint Conference on Artificial Intelligence (Buenos Aires), 41484152.

Bertin-Mahieux, T., Ellis, D. P., Whitman, B., and Lamere, P. (2011). The million song dataset. in Proceedings of the 12th International Society for Music Information Retrieval Conference, ISMIR 2011 (Miami, FL), 591-596.

Binas, J., Neil, D., Liu, S.-C., and Delbruck, T. (2017). DDD17: end-to-end DAVIS driving dataset. arXiv preprint arXiv:1711.01458.

Bojarski, M., Del Testa, D., Dworakowski, D., Firner, B., Flepp, B., Goyal, P., et al. (2016). End to end learning for self-driving cars. arXiv preprint arXiv:1604.07316.

Brandli, C., Berner, R., Yang, M., Liu, S. C., and Delbruck, T. (2014). A 240180130 $\mathrm{dB} 3 \mu \mathrm{s}$ latency global shutter spatiotemporal vision sensor. IEEE J. Solid-State Circuits 49, 2333-2341. doi: 10.1109/JSSC.2014.2342715

Brockman, G., Cheung, V., Pettersson, L., Schneider, J., Schulman, J., Tang, J., and Zaremba, W. (2016). OpenAI Gym. arXiv preprint arXiv:1606.01540.

Burgués, J., Jiménez-Soto, J. M., and Marco, S. (2018). Estimation of the limit of detection in semiconductor gas sensors through linearized calibration models. Analytica Chimica Acta 1013, 13-25. doi: 10.1016/j.aca.2018.01.062

Calabrese, E., Taverni, G., Easthope, C. A., Skriabine, S., Corradi, F., Longinotti, L., et al. (2019). "DHP19: Dynamic vision sensor 3D human pose dataset," in IEEE Computer Society Conference on Computer Vision and Pattern Recognition Workshops, Vol. 2019 (Long Beach, CA), 1695-1704.

Ceolini, E., Kiselev, I., and Liu, S. C. (2020). Evaluating multi-channel multidevice speech separation algorithms in the wild: a hardware-software solution. IEEE/ACM Trans. Audio Speech Lang. Process. 28, 1428-1439. doi: 10.1109/TASLP.2020.2989545

Chen, G., Cao, H., Aafaque, M., Chen, J., Ye, C., Röhrbein, F., et al. (2018a). Neuromorphic vision based multivehicle detection and tracking for intelligent transportation system. J. Adv. Transp. 2018, 1-13. doi: 10.1155/2018/4815383

Chen, Y., Wang, J., Li, J., Lu, C., Luo, Z., Xue, H., et al. (2018b). "LiDAR-video driving dataset: learning driving policies effectively," in Proceedings of the IEEE Computer Society Conference on Computer Vision and Pattern Recognition (Salt Lake City, UT), 5870-5878.

Chicca, E., Stefanini, F., Bartolozzi, C., and Indiveri, G. (2014). Neuromorphic electronic circuits for building autonomous cognitive systems. Proc. IEEE 102, 1367-1388. doi: 10.1109/JPROC.2014.2313954
Cohen, G., Afshar, S., Morreale, B., Bessell, T., Wabnitz, A., Rutten, M., et al. (2019). Event-based sensing for space situational awareness. J. Astron. Sci. 66, 125-141. doi: 10.1007/s40295-018-00140-5

Cohen, G., Afshar, S., Orchard, G., Tapson, J., Benosman, R., and Van Schaik, A. (2018). Spatial and temporal downsampling in event-based visual classification. IEEE Trans. Neural Netw. Learn. Syst. 29, 5030-5044. doi: 10.1109/TNNLS.2017.2785272

Conradt, J., Cook, M., Berner, R., Lichtsteiner, P., Douglas, R. J., and Delbruck, T. (2009). "A pencil balancing robot using a pair of AER dynamic vision sensors," in Proceedings - IEEE International Symposium on Circuits and Systems (Taipei: IEEE), 781-784.

Cordts, M., Omran, M., Ramos, S., Rehfeld, T., Enzweiler, M., Benenson, R., et al. (2016). "The cityscapes dataset for semantic urban scene understanding," in Proceedings of the IEEE Computer Society Conference on Computer Vision and Pattern Recognition, Vol. 2016 (Las Vegas, NV), 3213-3223.

D’Angelo, G., Janotte, E., Schoepe, T., O’Keeffe, J., Milde, M. B., Chicca, E., et al. (2020). Event-based eccentric motion detection exploiting time difference encoding. Front. Neurosci. 14:451. doi: 10.3389/fnins.2020.00451

Davies, M. (2019). Benchmarks for progress in neuromorphic computing. Nat. Mach. Intell. 1, 386-388. doi: 10.1038/s42256-019-0097-1

Davies, M., Srinivasa, N., Lin, T. H., Chinya, G., Cao, Y., Choday, S. H., et al. (2018). Loihi: a neuromorphic manycore processor with on-chip learning. IEEE Micro 38, 82-99. doi: 10.1109/MM.2018.112130359

Diehl, P. U., and Cook, M. (2015). Unsupervised learning of digit recognition using spike-timing-dependent plasticity. Front. Comput. Neurosci. 9:99. doi: 10.3389 /fncom.2015.00099

Dosovitskiy, A., Ros, G., Codevilla, F., Lopez, A., and Koltun, V. (2017). CARLA: an open urban driving simulator. arXiv preprint arXiv:1711.03938.

Dustdar, S., Mutlu, O., and Vijaykumar, N. (2020). Rethinking divide and conquertowards holistic interfaces of the computing stack. IEEE Internet Comput. 24, 45-57. doi: 10.1109/MIC.2020.3026245

Everingham, M., Eslami, S. M., Van Gool, L., Williams, C. K., Winn, J., and Zisserman, A. (2015). The pascal visual object classes challenge: a retrospective. Int. J. Comput. Vis. 111, 98-136. doi: 10.1007/s11263-014-0733-5

Falotico, E., Vannucci, L., Ambrosano, A., Albanese, U., Ulbrich, S., Tieck, J. C. V., et al. (2017). Connecting artificial brains to robots in a comprehensive simulation framework: The neurorobotics platform. Front. Neurorobot. 11:2. doi: 10.3389/fnbot.2017.00002

Fei-Fei, L., Fergus, R., and Perona, P. (2004). "Learning generative visual models from few training examples: an incremental bayesian approach tested on 101 object categories," in IEEE Computer Society Conference on Computer Vision and Pattern Recognition Workshops, Vol. 2004 (Washington, DC: IEEE), 178.

Fei-Fei, L., Fergus, R., and Perona, P. (2006). One-shot learning of object categories. IEEE Trans. Pattern Anal. Mach. Intell. 28, 594-611. doi: 10.1109/TPAMI.2006.79

Fenton, M. B. (1984). Echolocation: implications for ecology and evolution of bats. Quart. Rev. Biol. 59, 33-53. doi: 10.1086/413674

Finateu, T., Niwa, A., Matolin, D., Tsuchimoto, K., Mascheroni, A., Reynaud, E., et al. (2020). "0 a 1280-720 back-illuminated stacked temporal contrast eventbased vision sensor with $4.86 \mu \mathrm{m}$ Pixels, 1.066GEPS readout, programmable event-rate controller and compressive data-formatting pipeline," in Digest of Technical Papers - IEEE International Solid-State Circuits Conference, Vol. 2020 (San Francisco, CA: IEEE), 112-114.

Firoozian, R. (2014). "Feedback Control Theory Continues," in Servo Motors and Industrial Control Theory, Chapter 2 (Cham; Basel: Springer), 17-48. doi: 10.1007/978-3-319-07275-3

Flock, R., and Wersäll, J. (1962). A study of the orientation of the sensory hairs of the receptor cells in the lateral line organ of fish, with special reference to the function of the receptors. J. Cell Biol. 15, 19-27. doi: 10.1083/jcb.15.1.19

Foggia, P., Petkov, N., Saggese, A., Strisciuglio, N., and Vento, M. (2015). Reliable detection of audio events in highly noisy environments. Pattern Recognit. Lett. 65, 22-28. doi: 10.1016/j.patrec.2015.06.026

Fonollosa, J., Rodríguez-Luján, I., Trincavelli, M., Vergara, A., and Huerta, R. (2014). Chemical discrimination in turbulent gas mixtures with MOX sensors validated by gas chromatography-mass spectrometry. Sensors (Switzerland) 14, 19336-19353. doi: $10.3390 / \mathrm{s} 141019336$

Friston, K. (2010). The free-energy principle: a unified brain theory? Nat. Rev. Neurosci. 11, 127-138. doi: 10.1038/nrn2787 
Furber, S. (2016). "The SpiNNaker project," in Lecture Notes in Computer Science (including subseries Lecture Notes in Artificial Intelligence and Lecture Notes in Bioinformatics), Vol. 9726 (Berlin: Springer Nature), 652-665.

Gaidon, A., Wang, Q., Cabon, Y., and Vig, E. (2016). "VirtualWorlds as proxy for multi-object tracking analysis," in Proceedings of the IEEE Computer Society Conference on Computer Vision and Pattern Recognition, Vol. 2016 (Las Vegas, NV), 4340-4349.

Garofolo, J. S., Lamel, L. F., Fisher, W. M., Fiscus, J. G., Pallett, D. S., Dahlgren, N. L., et al. (1993). TIMIT Acoustic-Phonetic Continuous Speech Corpus. Philadelphia: Linguistic Data Consortium.

Gehrig, D., Gehrig, M., Hidalgo-Carrio, J., and Scaramuzza, D. (2020). "Video to events: recycling video datasets for event cameras," in Proceedings of the IEEE Computer Society Conference on Computer Vision and Pattern Recognition (Seattle, WA), 3583-3592.

Gehrig, M., Aarents, W., Gehrig, D., and Scaramuzza, D. (2021). DSEC: a stereo event camera dataset for driving scenarios. IEEE Robot. Autom. Lett. 6, 49474954. doi: 10.1109/LRA.2021.3068942

Geiger, A., Lenz, P., Stiller, C., and Urtasun, R. (2013). Vision meets robotics: the KITTI dataset. Int. J. Robot. Res. 32, 1231-1237. doi: $10.1177 / 0278364913491297$

Geirhos, R., Janssen, D. H. J., Schütt, H. H., Rauber, J., Bethge, M., and Wichmann, F. A. (2017). Comparing deep neural networks against humans: object recognition when the signal gets weaker. arXiv preprint arXiv:1706.06969.

Gemmeke, J. F., Ellis, D. P. W., Freedman, D., Jansen, A., Lawrence, W., Moore, R. C., et al. (2017). "Audio set: an ontology and human-labeled dataset for audio events," in 2017 IEEE International Conference on Acoustics, Speech and Signal Processing (ICASSP) (New Orleans, LA: IEEE), 776-780.

Gini, F., and Rangaswamy, M. (2008). Knowledge Based Radar Detection, Tracking and Classification, Vol. 52. Hoboken, NJ: John Wiley \& Sons.

Griffin, D. R. (1958). Listening in the Dark: the Acoustic Orientation of Bats and Men. New Haven, CT: Yale University Press.

Griffin, G., Holub, A., and Perona, P. (2007). Caltech-256 object category dataset. Caltech Mimeo 11, 20.

Grother, P. (1995). (Latin letters) NIST special database 19 handprinted forms and characters database. doi: 10.18434/T4H01C

Hamilton, T. J., Afshar, S., Van Schaik, A., and Tapson, J. (2014). Stochastic electronics: a neuro-inspired design paradigm for integrated circuits. Proc. IEEE 102, 843-859. doi: 10.1109/JPROC.2014.2310713

He, K., Zhang, X., Ren, S., and Sun, J. (2016). "Deep residual learning for image recognition," in Proceedings of the IEEE Computer Society Conference on Computer Vision and Pattern Recognition, Vol. 2016 (Las Vegas, NV), 770-778.

Heittola, T., Mesaros, A., and Virtanen, T. (2020). Acoustic scene classification in DCASE 2020 challenge: generalization across devices and low complexity solutions. arXiv preprint arXiv:2005.14623.

Hofmann, V., Sanguinetti-Scheck, J. I., Künzel, S., Geurten, B., Gómez-Sena, L., and Engelmann, J. (2013). Sensory flow shaped by active sensing: sensorimotor strategies in electric fish. J. Exp. Biol. 216, 2487-2500. doi: 10.1242/jeb.082420

Hogendoorn, H., and Burkitt, A. N. (2018). Predictive coding of visual object position ahead of moving objects revealed by time-resolved EEG decoding. NeuroImage 171, 55-61. doi: 10.1016/j.neuroimage.2017.12.063

Hu, Y., Binas, J., Neil, D., Liu, S. C., and Delbruck, T. (2020). “DDD20 end-to-end event camera driving dataset: fusing frames and events with deep learning for improved steering prediction," in 2020 IEEE 23rd International Conference on Intelligent Transportation Systems, ITSC 2020 (Rhodes).

Hu, Y., Liu, H., Pfeiffer, M., and Delbruck, T. (2016). DVS benchmark datasets for object tracking, action recognition, and object recognition. Front. Neurosci. 10:405. doi: $10.3389 /$ fnins.2016.00405

Hu, Y., Liu, S. C., and Delbruck, T. (2021). "V2e: from video frames to realistic DVS events," in IEEE Computer Society Conference on Computer Vision and Pattern Recognition Workshops (Nashville, TN), 1312-1321.

Jackson, Z., Souza, C., Flaks, J., Pan, Y., Nicolas, H., and Thite, A. (2018). Jakobovski/free-spoken-digit-dataset: v1.0.8. doi: 10.5281/zenodo.1342401

Jaderberg, M., Czarnecki, W. M., Dunning, I., Marris, L., Lever, G., Castañeda, A. G., et al. (2019). Human-level performance in 3D multiplayer games with population-based reinforcement learning. Science 364, 859-865. doi: $10.1126 /$ science.aau6249

Jakobi, N., Husbands, P., and Harvey, I. (1995). Noise and the reality gap: The use of simulation in evolutionary robotics. Lecture Notes in Computer Science (including subseries Lecture Notes in Artificial Intelligence and Lecture Notes in Bioinformatics) (Berlin: Springer-Verlag), Vol. 929, 704-720.

Jia Deng, Wei Dong, Socher, R., Li-Jia Li, Kai Li, and Li Fei-Fei (2009). "ImageNet: a large-scale hierarchical image database," In 2009 IEEE Conference on computer vision and Pattern Recognition (Miami, FL: IEEE), 248-255.

Jolley, A., Cohen, G., Joubert, D., and Lambert, A. (2021). Evaluation of eventbased sensors for satellite material characterization. J. Spacecraft Rockets 1-10. doi: 10.2514/1.A35015

Jordan, J., Weidel, P., and Morrison, A. (2019). A closed-loop toolchain for neural network simulations of learning autonomous agents. Front. Comput. Neurosci. 13:46. doi: 10.3389/fncom.2019.00046

Joubert, D., Marcireau, A., Ralph, N., Jolley, A., van Schaik, A., and Cohen, G. (2021). Event camera simulator improvements via characterized parameters. Front. Neurosci. 15:910. doi: 10.3389/fnins.2021.702765

Kaiser, J., Tieck, J. C., Hubschneider, C., Wolf, P., Weber, M., Hoff, M., et al. (2017). "Towards a framework for end-to-end control of a simulated vehicle with spiking neural networks," in 2016 IEEE International Conference on Simulation, Modeling, and Programming for Autonomous Robots, SIMPAR 2016 (San Francisco, CA: IEEE), 127-134.

Kay, W., Carreira, J., Simonyan, K., Zhang, B., Hillier, C., Vijayanarasimhan, S., et al. (2017). The kinetics human action video dataset. arXiv preprint arXiv: 1705.06950 .

Keller, G. B., and Mrsic-Flogel, T. D. (2018). Predictive processing: a canonical cortical computation. Neuron 100, 424-435. doi: 10.1016/j.neuron.2018.10.003

Koenig, N., and Howard, A. (2004). "Design and use paradigms for Gazebo, an open-source multi-robot simulator," in 2004 IEEE/RSJ International Conference on Intelligent Robots and Systems (IROS), Vol. 3 (Sendai), 2149-2154.

Koizumi, Y., Saito, S., Uematsu, H., Harada, N., and Imoto, K. (2019). "ToyADMOS: a dataset of miniature-machine operating sounds for anomalous sound detection," in IEEE Workshop on Applications of Signal Processing to Audio and Acoustics, Vol. 2019 (New Paltz, NY: IEEE), 313-317.

Krizhevsky, A., Sutskever, I., and Hinton, G. E. (2017). ImageNet classification with deep convolutional neural networks. Commun. ACM 60, 84-90. doi: 10.1145/3065386

LeCun, Y., Bottou, L., Bengio, Y., and Haffner, P. (1998). Gradient-based learning applied to document recognition. Proc. IEEE 86, 2278-2323. doi: $10.1109 / 5.726791$

Leonard, R. G., and Doddington, G. (1993). Tidigits Speech Corpus. Philadelphia, PA: Linguistic Data Consortium.

Liang, D., and Indiveri, G. (2019). A neuromorphic computational primitive for robust context-dependent decision making and context-dependent stochastic computation. IEEE Trans. Circuits Syst. II Exp. Briefs 66, 843-847. doi: 10.1109/TCSII.2019.2907848

Lichtsteiner, P., Posch, C., and Delbruck, T. (2008). A 128 -128 120 dB 15 $\mu \mathrm{s}$ latency asynchronous temporal contrast vision sensor. IEEE J. Solid-State Circuits 43, 566-576. doi: 10.1109/JSSC.2007.914337

Lin, T. Y., Maire, M., Belongie, S., Hays, J., Perona, P., Ramanan, D., et al. (2014). "Microsoft COCO: common objects in context," in Lecture Notes in Computer Science (including subseries Lecture Notes in Artificial Intelligence and Lecture Notes in Bioinformatics) LNCS(PART 5), Vol. 8693 (Cham; Basel: Springer), 740-755.

Liu, S. C., and Delbruck, T. (2010). Neuromorphic sensory systems. Curr. Opin. Neurobiol. 20, 288-295. doi: 10.1016/j.conb.2010.03.007

Lungu, I. A., Corradi, F., and Delbruck, T. (2017). Live demonstration: convolutional neural network driven by dynamic vision sensor playing RoShamBo. In Proceedings - IEEE International Symposium on Circuits and Systems (Baltimore, MD: IEEE), 1.

Madaan, R., Gyde, N., Vemprala, S., Brown, M., Nagami, K., Taubner, T., et al. (2020). AirSim drone racing lab. arXiv preprint arXiv:2003.05654.

Maddern, W., Pascoe, G., Linegar, C., and Newman, P. (2017). 1 year, $1000 \mathrm{~km}$ : the oxford robotcar dataset. Int. J. Robot. Res. 36, 3-15. doi: $10.1177 / 0278364916679498$

Masquelier, T. (2012). Relative spike time coding and STDP-based orientation selectivity in the early visual system in natural continuous and saccadic vision: a computational model. J. Comput. Neurosci. 32, 425-441. doi: 10.1007/s10827-011-0361-9

Mead, C. (1990). Neuromorphic electronic systems. Proc. IEEE 78, 1629-1636. doi: $10.1109 / 5.58356$ 
Miao, S., Chen, G., Ning, X., Zi, Y., Ren, K., Bing, Z., et al. (2019). Neuromorphic vision datasets for pedestrian detection, action recognition, and fall detection. Front. Neurorobot. 13:38. doi: 10.3389/fnbot.2019.00038

Milde, M. B. (2019). Spike-Based Computational Primitives for Vision-Based Scene Understanding. Ph.D. thesis, University of Zurich.

Milde, M. B., Blum, H., Dietmüller, A., Sumislawska, D., Conradt, J., Indiveri, G., and Sandamirskaya, Y. (2017). Obstacle avoidance and target acquisition for robot navigation using a mixed signal analog/digital neuromorphic processing system. Front. Neurorobot. 11:28. doi: 10.3389/fnbot.2017.00028

Mnih, V., Kavukcuoglu, K., Silver, D., Graves, A., Antonoglou, I., Wierstra, D., et al. (2013). Playing atari with deep reinforcement learning. arXiv preprint arXiv:1312.5602.

Moeys, D. P., Corradi, F., Kerr, E., Vance, P., Das, G., Neil, D., et al. (2016). "Steering a predator robot using a mixed frame/event-driven convolutional neural network," in 2016 2nd International Conference on Event-Based Control, Communication, and Signal Processing, EBCCSP 2016 - Proceedings (Krakw: IEEE), 1-8.

Moradi, S., Qiao, N., Stefanini, F., and Indiveri, G. (2018). A scalable multicore architecture with heterogeneous memory structures for dynamic neuromorphic asynchronous processors (DYNAPs). IEEE Trans. Biomed. Circuits Syst. 12, 106-122. doi: 10.1109/TBCAS.2017.2759700

Mueggler, E., Rebecq, H., Gallego, G., Delbruck, T., and Scaramuzza, D. (2017). The event-camera dataset and simulator: event-based data for pose estimation, visual odometry, and SLAM. Int. J. Robot. Res. 36, 142-149. doi: 10.1177/0278364917691115

Nagrani, A., Chung, J. S., Xie, W., and Zisserman, A. (2020). Voxceleb: Largescale speaker verification in the wild. Comput. Speech Lang. 60:101027. doi: 10.1016/j.csl.2019.101027

OpenAI:, Berner, C., Brockman, G., Chan, B., Cheung, V., Dębiak, P., et al. (2019). Dota 2 with large scale deep reinforcement learning. arXiv preprint arXiv:1912.06680.

Orchard, G., Jayawant, A., Cohen, G. K., and Thakor, N. (2015a). Converting static image datasets to spiking neuromorphic datasets using saccades. Front. Neurosci. 9.

Orchard, G., Meyer, C., Etienne-Cummings, R., Posch, C., Thakor, N., and Benosman, R. (2015b). HFirst: a temporal approach to object recognition. IEEE Trans. Pattern Anal. Mach. Intell. 37, 2028-2040. doi: 10.1109/TPAMI.2015.2392947

Park, P. K., Soloveichik, E., Ryu, H. E., Seok Kim, J., Shin, C. W., Lee, H., et al. (2019). "Low-latency interactive sensing for machine vision," in Technical Digest - International Electron Devices Meeting, IEDM, Vol. 2019 (San Francisco, CA: IEEE), 10-16.

Pérez-Carrasco, J. A., Zhao, B., Serrano, C., Acha, B., Serrano-Gotarredona, T., Chen, S., et al. (2013). Mapping from frame-driven to frame-free eventdriven vision systems by low-rate rate coding and coincidence processing Application to feedforward convnets. IEEE Trans. Pattern Anal. Mach. Intell. 35, 2706-2719. doi: 10.1109/TPAMI.2013.71

Perot, E., de Tournemire, P., Nitti, D., Masci, J., and Sironi, A. (2020). "Learning to detect objects with a 1 megapixel event camera," in Advances in Neural Information Processing Systems (Vancouver, CA: NeurIPS).

Politis, A., Adavanne, S., and Virtanen, T. (2020). A dataset of reverberant spatial sound scenes with moving sources for sound event localization and detection. arXiv preprint arXiv:2006.01919.

Pradhan, B. R., Bethi, Y., Narayanan, S., Chakraborty, A., and Thakur, C. S. (2019). "N-HAR: a neuromorphic event-based human activity recognition system using memory surfaces," in Proceedings - IEEE International Symposium on Circuits and Systems, Vol. 2019 (Sapporo: IEEE), 1-5.

Qiao, N., Mostafa, H., Corradi, F., Osswald, M., Stefanini, F., Sumislawska, D., and Indiveri, G. (2015). A reconfigurable on-line learning spiking neuromorphic processor comprising 256 neurons and 128K synapses. Front. Neurosci. 9:141. doi: 10.3389/fnins.2015.00141

Quigley, M., Conley, K., Gerkey, B., Faust, J., Foote, T., Leibs, J., Wheeler, R., and Ng, A. Y. (2009). "ROS: an open-source Robot Operating System," in ICRA Workshop on Open Source Software, Vol. 3.2 (Kobe), 5.

Rao, R. P. N., and Ballard, D. H. (1999). Predictive coding in the visual cortex: a functional interpretation of some extra-classical receptive-field effects. Nat. Neurosci. 2, 79-87. doi: 10.1038/4580
Rasmussen, D., Voelker, A., and Eliasmith, C. (2017). A neural model of hierarchical reinforcement learning. PLoS ONE 12:e0180234. doi: 10.1371 /journal.pone.0180234

Rebecq, H., Gehrig, D., and Scaramuzza, D. (2018). "ESIM: an open event camera simulator," in Conference on Robot Learning (Zürich: PMLR), 969-982.

Rebecq, H., Ranftl, R., Koltun, V., and Scaramuzza, D. (2019). "Events-to-video: bringing modern computer vision to event cameras," in Proceedings of the IEEE Computer Society Conference on Computer Vision and Pattern Recognition, Vol. 2019 (Long Beach, CA), 3852-3861.

Rees, W. G. (1990). Physical Principles of Remote Sensing. Cambridge, UK: Cambridge University Press.

Remy, I. (2019). Power and Area Optimization of a Dynamic Vision Sensor in $65 \mathrm{~nm}$ CMOS. (Master's thesis). Université Catholique de Louvain.

Rothe, R., Timofte, R., and Van Gool, L. (2018). Deep expectation of real and apparent age from a single image without facial landmarks. Int. J. Comput. Vis. 126, 144-157. doi: 10.1007/s11263-016-0940-3

Rueckauer, B., and Delbruck, T. (2016). Evaluation of event-based algorithms for optical flow with ground-truth from inertial measurement sensor. Front. Neurosci. 10:176. doi: 10.3389/fnins.2016.00176

Samsung (2020). Samsung SmartThings Vision. available online at: https://www.samsung.com/au/smartthings/camera/smartthings-visiongp-u999gteeaac/

Santana, E., and Hotz, G. (2016). Learning a driving simulator. arXiv preprint arXiv:1608.01230.

Scheerlinck, C., Rebecq, H., Stoffregen, T., Barnes, N., Mahony, R., and Scaramuzza, D. (2019). "CED: color event camera dataset," in IEEE Computer Society Conference on Computer Vision and Pattern Recognition Workshops, Volume 2019 (Long Beach, CA), 1684-1693.

Schmuker, M., and Schneider, G. (2007). Processing and classification of chemical data inspired by insect olfaction. Proc. Natl. Acad. Sci. U.S.A. 104, 20285-20289. doi: 10.1073/pnas.0705683104

Schneider, P., and Schneider, G. (2003). Collection of bioactive reference compounds for focused library design. QSAR Combinatorial Sci. 22, 713-718. doi: 10.1002/qsar.200330825

Segata, M., and Cigno, R. L. (2019). Automatic Emergency Braking With Pedestrian Detection. Technical Report, American Automobile Association.

Serrano-Gotarredona, T., and Linares-Barranco, B. (2015). Poker-DVS and MNIST-DVS. Their history, how they were made, and other details. Front. Neurosci. 9:481. doi: 10.3389/fnins.2015.00481

Shalev-Shwartz, S. (2011). Online learning and online convex optimization. Found. Trends Mach. Learn. 4, 107-194. doi: 10.1561/2200000018

Silver, D., Hubert, T., Schrittwieser, J., Antonoglou, I., Lai, M., Guez, A., et al. (2018). A general reinforcement learning algorithm that masters chess, shogi, and Go through self-play. Science 362, 1140-1144. doi: 10.1126/science.aar6404

Silver, D., Schrittwieser, J., Simonyan, K., Antonoglou, I., Huang, A., Guez, A., et al. (2017). Mastering the game of Go without human knowledge. Nature 550, 354-359. doi: 10.1038/nature24270

Smaira, L., Carreira, J., Noland, E., Clancy, E., Wu, A., and Zisserman, A. (2020). A short note on the kinetics-700-2020 human action dataset. arXiv preprint arXiv:2010.10864.

Stewart, T. C., DeWolf, T., Kleinhans, A., and Eliasmith, C. (2015). Closed-loop neuromorphic benchmarks. Front. Neurosci. 9:464. doi: 10.3389/fnins.2015.00464

Stock, P., and Cisse, M. (2018). "ConvNets and imagenet beyond accuracy: understanding mistakes and uncovering biases," in Lecture Notes in Computer Science (including subseries Lecture Notes in Artificial Intelligence and Lecture Notes in Bioinformatics) LNCS, Vol. 11210 (Cham, Basel: Springer), 504-519.

Tan, C., Lallee, S., and Orchard, G. (2015). Benchmarking neuromorphic vision: lessons learnt from computer vision. Front. Neurosci. 9:374. doi: $10.3389 /$ fnins.2015.00374

Thakur, C. S., Molin, J. L., Cauwenberghs, G., Indiveri, G., Kumar, K., Qiao, N., et al. (2019). Corrigendum: large-scale neuromorphic spiking array processors: a quest to mimic the brain. Front. Neurosci. 12:891. doi: 10.3389/fnins.2018.00991

Torralba, A., and Efros, A. A. (2011). "Unbiased look at dataset bias," in Proceedings of the IEEE Computer Society Conference on Computer Vision and Pattern Recognition (Colorado Springs, CO), 1521-1528. 
Torralba, A., Fergus, R., and Freeman, W. T. (2008). 80 million tiny images: a large data set for nonparametric object and scene recognition. IEEE Trans. Pattern Anal. Mach. Intell. 30, 1958-1970. doi: 10.1109/TPAMI.20 08.128

Vergara, A., Fonollosa, J., Mahiques, J., Trincavelli, M., Rulkov, N., and Huerta, R. (2013). On the performance of gas sensor arrays in open sampling systems using inhibitory support vector machines. Sens. Actuat. B Chem. 185, 462-477. doi: 10.1016/j.snb.2013.05.027

Vinyals, O., Babuschkin, I., Czarnecki, W. M., Mathieu, M., Dudzik, A., Chung, J., et al. (2019). Grandmaster level in StarCraft II using multiagent reinforcement learning. Nature 575, 350-354. doi: 10.1038/s41586-019$1724-z$

Wang, Z., She, Q., and Ward, T. E. (2021). Generative adversarial networks in computer vision: a survey and taxonomy. ACM Comput. Surveys 54, 1-38. doi: $10.1145 / 3439723$

Xiao, H., Rasul, K., and Vollgraf, R. (2017). Fashion-MNIST: a novel image dataset for benchmarking machine learning algorithms. arXiv preprint arXiv: 1708.07747

Xu, C. S., Januszewski, M., Lu, Z., Takemura, S.-y., Hayworth, K., Huang, G., et al. (2020). A connectome of the adult drosophila central brain. BioRxiv.

Yang, M., Liu, S. C., and Delbruck, T. (2017). Analysis of encoding degradation in spiking sensors due to spike delay variation. IEEE Trans. Circuits Syst. I Reg. Papers 64, 145-155. doi: 10.1109/TCSI.2016.2613503

Yousefzadeh, A., Orchard, G., Serrano-Gotarredona, T., and Linares-Barranco, B. (2018). Active perception with dynamic vision sensors. minimum saccades with optimum recognition. IEEE Trans. Biomed. Circuits Syst. 12, 927-939. doi: 10.1109/TBCAS.2018.2834428

Yu, F., Chen, H., Wang, X., Xian, W., Chen, Y., Liu, F., et al. (2020). "BDD100K: a diverse driving dataset for heterogeneous multitask learning," in 2020 IEEE/CVF Conference on Computer Vision and Pattern Recognition (CVPR) (Seattle, WA: IEEE), 2633-2642.
Zhao, B., Ding, R., Chen, S., Linares-Barranco, B., and Tang, H. (2015). Feedforward categorization on aer motion events using cortex-like features in a spiking neural network. IEEE Trans. Neural Netw. Learn. Syst. 26, 1963-1978. doi: 10.1109/TNNLS.2014.2362542

Zhu, A. Z., Thakur, D., Özaslan, T., Pfrommer, B., Kumar, V., and Daniilidis, K. (2018). The multivehicle stereo event camera dataset: an event camera dataset for 3D perception. IEEE Robot. Autom. Lett. 3, 2032-2039. doi: 10.1109/LRA.2018.2800793

Ziyatdinov, A., Fonollosa, J., Fernández, L., Gutierrez-Gálvez, A., Marco, S., and Perera, A. (2015). Bioinspired early detection through gas flow modulation in chemo-sensory systems. Sens. Actuat. B Chem. 206, 538-547. doi: 10.1016/j.snb.2014.09.001

Conflict of Interest: The authors declare that the research was conducted in the absence of any commercial or financial relationships that could be construed as a potential conflict of interest.

Publisher's Note: All claims expressed in this article are solely those of the authors and do not necessarily represent those of their affiliated organizations, or those of the publisher, the editors and the reviewers. Any product that may be evaluated in this article, or claim that may be made by its manufacturer, is not guaranteed or endorsed by the publisher.

Copyright (๑) 2022 Milde, Afshar, Xu, Marcireau, Joubert, Ramesh, Bethi, Ralph, El Arja, Dennler, van Schaik and Cohen. This is an open-access article distributed under the terms of the Creative Commons Attribution License (CC BY). The use, distribution or reproduction in other forums is permitted, provided the original author(s) and the copyright owner(s) are credited and that the original publication in this journal is cited, in accordance with accepted academic practice. No use, distribution or reproduction is permitted which does not comply with these terms. 